\title{
INTENSITY INTERFEROMETRY FOR THE 21ST CENTURY
}

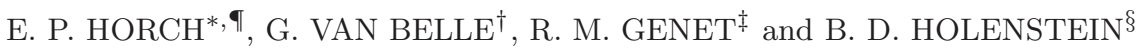 \\ * Department of Physics \\ Southern Connecticut State University \\ New Haven, CT 06515, USA \\ horche2@sourthernct.edu \\ ${ }^{\dagger}$ Lowell Observatory \\ 1400 W Mars Hill Road \\ Flagstaff, AZ 86001, USA \\ gerard@lowell.edu \\ ${ }^{\ddagger}$ California Polytechnic State University \\ One Grand Avenue, San Luis Obispo \\ CA 93407, USA \\ russmgenet@aol.com

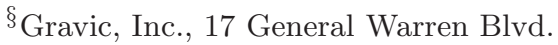 \\ Malvern, PA 19355, USA \\ 『Adjunct Astronomer \\ Lowell Observatory
}

Received 2013 June 30; Revised 2013 October 20; Accepted 2013 November 5; Published 2014 January 6

\begin{abstract}
Advances in detector technology and electronic timing capabilities in recent years have resulted in a new opportunity for ultra-high resolution in astronomy using intensity interferometry. We have been working with this technology and describe here the potential as we see it. Two separate opportunities exist at present: the use of Single Photon Avalanche Diode (SPAD) detectors with existing researchgrade telescopes and photomultipliers coupled with light bucket telescopes. In the future, there may also be potential for space-based intensity interferometry. While intensity interferometry is not likely to replace amplitude-based interferometry, it does have certain advantages in terms of portability, use of large baselines, narrow-band imaging, and imaging in the blue. We see a new possibility for its use particularly in stellar astrophysics for these reasons.
\end{abstract}

Keywords: Instrumentation: interferometers, techniques: interferometric, high angular resolution.

\section{Introduction}

It has been known since the 1950s that in addition to phase correlations that exist in the detected light from a source, intensity correlations also exist, although at lower signal-to-noise ratio (Hanbury Brown \& Twiss, 1956). These correlated intensity fluctuations are essentially related to beat frequencies between photons of nearly the same frequency that arrive at pairs of stations that make up the interferometer. If the filter bandwidth is kept very narrow, then these beat frequencies can be significantly lower than the frequency of the radiation itself, making them easier to detect. Given the properties of photodetectors of the 1950s, very large collectors were needed to observe even the brightest stars with this technique. Nonetheless, starting in the 1960s and continuing into the 1970s, Hanbury Brown and his colleagues operated a two-telescope stellar intensity interferometer, where each telescope had a $6.5-\mathrm{m}$ diameter aperture. The instrument successfully measured the diameters of 32 stars (Hanbury Brown, Davis \& Allen, 1974).

Despite inherent signal-to-noise limitations, we suggest that intensity interferometry deserves 
further study today as a viable technique for astronomical long baseline optical interferometry. There are essentially two reasons for this: (i) modern detectors and electronics have much better timing resolution and quantum efficiency than in the 1970s, and (ii) the technique is in principle passive, i.e. one can simply detect the stream of photon arrival times at each station and correlate these signals after the fact. This makes long baseline optical interferometry more akin to the radio regime - a regime where extremely large baselines can be used by combining the recorded signals from independent stations.

The University of Utah group has been working on a modern intensity interferometer in the context of air Cerenkov arrays, which essentially use "light bucket" telescopes (i.e. those not designed with high imaging quality in mind) and modern photomultiplier detectors (Lebohec et al., 2010; Nuñez et al., 2012). We are also interested in this approach; see e.g. Genet et al. (2013) for a recent description. In addition, we suggest an alternative approach based on Single Photon Avalanche Diode (SPAD) detectors. Commercially available SPAD detectors have quantum efficiencies of $\sim 0.5$. Regardless of the choice of detector, modern signal correlators, such as the PicoQuant Picoharp 300, have the ability to record and correlate data at bandwidths in excess of $\sim 10 \mathrm{GHz}$. The signal-to-noise ratio (SNR) in intensity interferometry in the shot-noise limited regime is given in e.g. Klein, Guelman, and Lipson (2007) by

$$
\frac{S}{N}=2.512^{-m} F_{0} A \eta\left|\gamma_{12}(B)\right|^{2} \sqrt{\frac{\Delta f T_{0}}{2}},
$$

where $m$ is the magnitude of the star in the wavelength observed, $F_{0}$ is the photon flux of a zero-magnitude star, $A$ is the area of a collector, $\eta$ is the optical system efficiency (that is, the optical and atmospheric transmission multiplied by the quantum efficiency of the detector), $B$ is the baseline, $\gamma_{12}$ is magnitude of the degree of coherence, $\Delta f$ is the detector bandwidth, and $T_{0}$ is the integration time. (For high light levels, the SNR is limited by wave noise as shown in e.g. Radhakrishnan, 1999). Using this formula, one can show that modern detector bandwidths and quantum efficiencies result in a gain in signal-tonoise versus the 1970s of over 60, which means that if the same aperture size were used today with this instrumentation, its limiting magnitude would be 4.5 magnitudes fainter, or about $\mathrm{V} \sim 7$. If the timing information is recorded with $\sim 10 \mathrm{GHz}$ bandwidth, then the optical paths in a given aperture must be equalized to about $1.5 \mathrm{~cm}$, a more stringent limit than in the 1970s; this limit can generally be met with telescopes with high optical quality but could pose a limitation for light bucket telescopes. Alternatively, the improvement in quantum efficiency and timing permits an interferometer with the limiting magnitude comparable to that of Hanbury Brown to be miniaturized, i.e. each telescope could be a factor of over 60 smaller, or in other words, as small as $\sim 80 \mathrm{~cm}$ in diameter. Intensity interferometry can therefore be done with many existing telescopes with sizes down to the $\sim 1-\mathrm{m}$ class. Using the above formula, Fig. 1 shows the observation time needed to reach a SNR of 5 for the correlation peak as a function of target magnitude for three situations: two 25-inch Dobsonian telescopes, readily available on the amateur market for $\sim \$ 20,000$ each (labeled SCSU in the figure, since this is an observing situation we have proposed for campus observing at Southern Connecticut State University), the 1.0-m and 1.8-m telescopes at Anderson Mesa, near Flagstaff, Arizona (i.e. Lowell Observatory, where one of us (Horch) has already conducted intensity interferometry observations which will be discussed in Sec. 3), and two 4-m class telescopes, such as the WIYN and Mayall telescopes at Kitt Peak (labeled KPNO in the figure below).

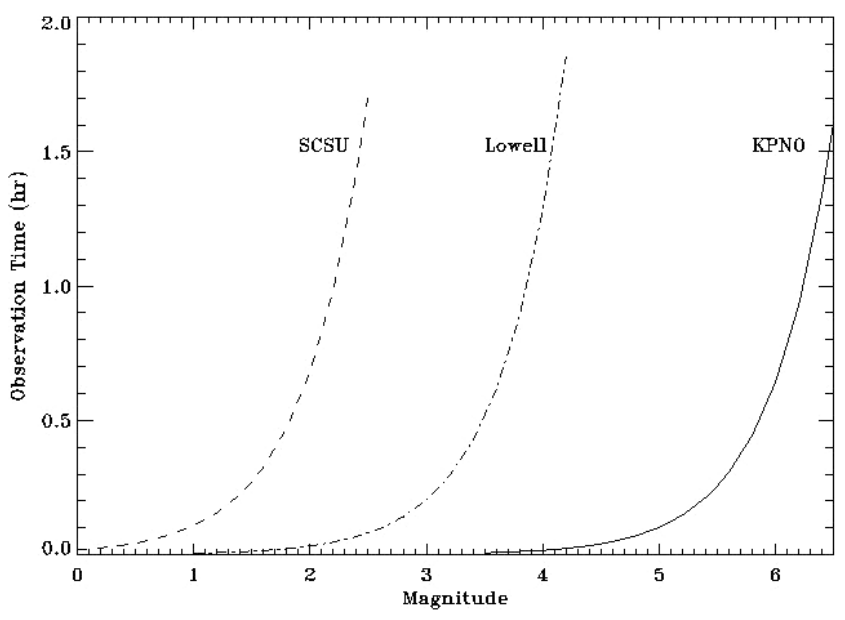

Fig. 1. Observation time required in order to obtain a signal-to-noise ratio of 5 as a function of $\mathrm{V}$ magnitude for the three two-station intensity interferometers discussed in the text. With more stations, these curves would shift to the right; see Sec. 4. 


\section{Intensity Interferometry versus Michelson Interferometry}

Michelson interferometry and intensity interferometry are the two main forms of interferometry that have been employed observationally in the visible, each notably with "long baselines" (at two or more independently pointed collecting apertures that are physically separated). Of the two techniques, the latter was employed first by Hanbury Brown and Twiss, starting with the demonstration of signal correlation detection on Sirius in 1956 as mentioned above, and culminating with the Narrabri Intensity Interferometer in the 1960s and 1970s, with its seminal measures on hot star stellar diameters. However, development of high-speed, high-dynamic range "delay lines" in the late 1970s allowed implementation of Michelson interferometers such as the Mark III to eclipse further use of intensity interferometers (Shao \& Staelin, 1977; Shao et al., 1986).

Michelson interferometers collect starlight from two or more apertures and typically equalize path lengths to within the coherence length (typically a fraction of the observing wavelength, depending on filter width and integration time), and then recombine the beams, allowing measurement of the interference fringes. Intensity interferometers correlate the fluctuations in intensity of light received at photoelectric detectors at the collecting apertures. Two-aperture versions of both interferometers allow for simple stellar size measurements. Historically, the principal attraction of Michelson interferometry over intensity interferometry has been sensitivity, while for intensity interferometry over Michelson, simplicity, particularly for the optical systems.

\section{Initial SPAD-Based Observing}

An approach that one of us (Horch) has pursued has been the use of SPAD devices at existing telescopes. The instrumentation consists of a PicoQuant Picoharp 300 timing correlator and two SPAD detectors from Micro Photon Devices (MPD), as well as two focal reducer packages made in the Physics Department machine shop at SCSU. Except for the focal reducers, the rest of the instrumentation fits easily in a carry-on suitcase. We have taken this set-up to Lowell Observatory on two occasions, December 2011 and June 2012, and placed a SPAD and focal reducer on the 1.8-m
Perkins telescope and the 1.0-m Hall telescope. (These are separated by about $50 \mathrm{~m}$ on Anderson Mesa.) Between the two domes, we set up an observing station consisting of a laptop computer to record data and the Picoharp correlator. Long cables were used to get the detector signals from the telescopes to the timing correlator module. The two telescopes, configured as an intensity interferometer, are shown in Fig. 2. Further information about the technical aspects of this experiment can be found in Horch and Camarata (2012).

These observing experiences have taught us that SPAD detectors do have three limitations that are relevant for astronomical observing. The first is small size. For example, MPD offers three sizes: 20-micron diameter active area, 50-micron, and 100-micron. Our devices are of the 50-micron type. Therefore, the focused spot size must be small. One cannot simply use a large collector with a large focus spot (i.e. a "light bucket" telescope) for these single-pixel detectors. To use the SPAD for this application, astronomical-grade optics must be employed to get as much of the light as possible onto the detector. This requirement is in theory easily met with many existing astronomical research telescopes. However in practice, even with the focal reducers, it was very difficult to point and focus the telescopes at Lowell in such a way as to maximize the signal.

Another limitation is dead time. The electronic signal of the MPD devices is produced with a timing uncertainty relative to the arrival time of the incoming photon of $\sim 50 \mathrm{ps}$, for an effective bandwidth in the $10 \mathrm{GHz}$ range, but the device is unresponsive to light for a period of $\sim 80 \mathrm{~ns}$ after this detection. Therefore, the maximum count rates that can be detected are only 10-12 MHz. This has the consequence of having a rather narrow "Goldilocks" zone of target brightness that can be observed with a given optical system and filter width: if the source is too bright, then the maximum count rate will limit the observation, and if the source is too faint, then there will be too few photons to correlate in a reasonable amount of time. The problem on the bright end can always be addressed with the use of a narrower filter, but perhaps a solution to both this problem and the first one mentioned above is the ongoing development of SPAD arrays rather than single-pixel devices. This would allow the light to be spread over a larger area, and therefore the photon flux on any one pixel would be reduced leading to a higher overall maximum count rate 

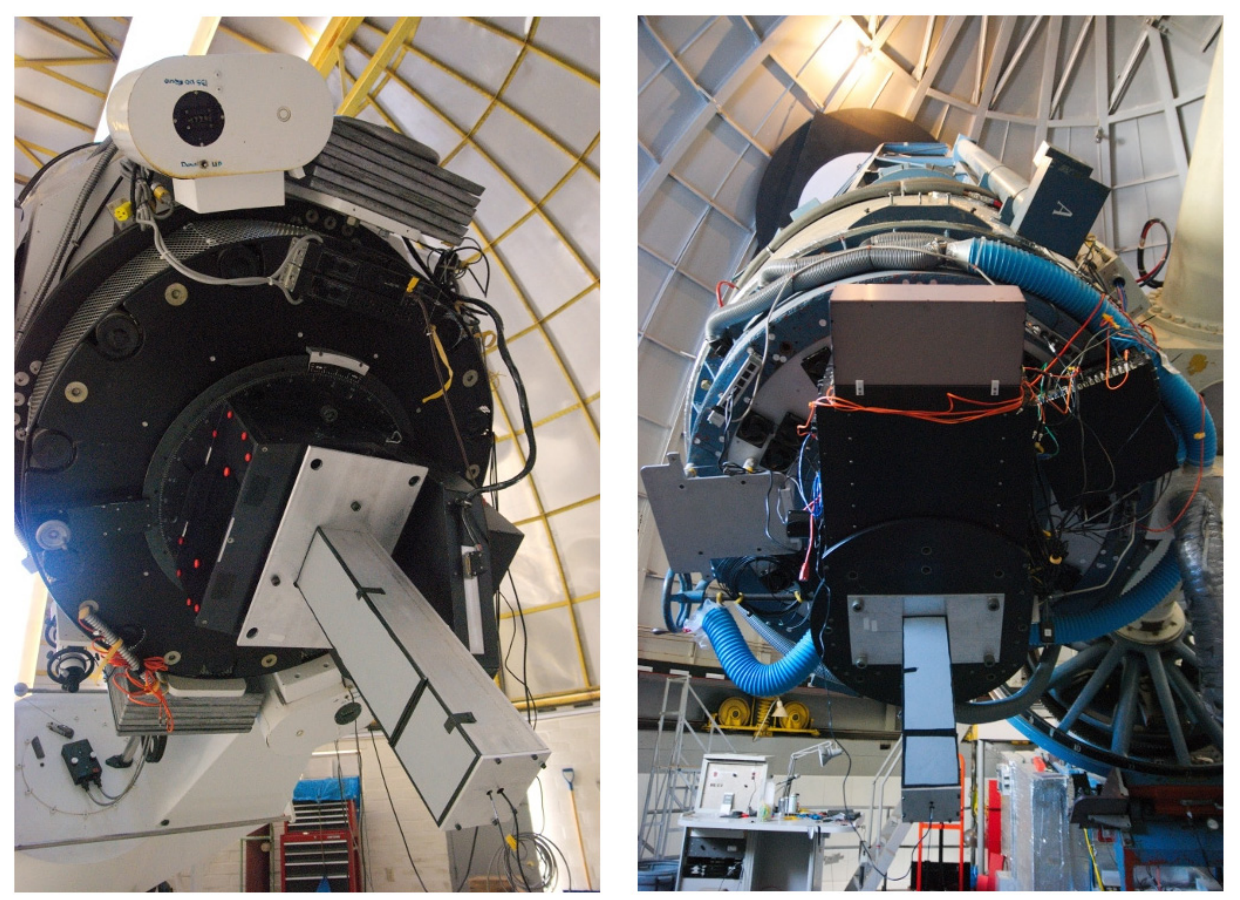

Fig. 2. The observing set-up at the 1.0-m (left) and 1.8-m (right) telescopes at Anderson Mesa, Lowell Observatory, respectively. In both cases, the focal reducer package is shown mounted at the Cassegrain focus of each telescope. The SPAD detectors sit in the lower portion of the focal reducer housing, and the signal cable runs to a location in between the two domes, where the Picoharp module is located.

would be achieved. Placing a lenslet over the SPAD would also help to increase the amount of signal detected.

The third limitation appears to be related to temperature. During the December run, the detectors would shut off after a period of time, typically about an hour after being turned on. This is apparently due to the low temperatures in the domes: the MPD housing tries to maintain a constant temperature on the device, and if the ambient temperature is either too high or too low, then a temperature lock cannot be maintained and the device powers off. In the lab, this obviously does not occur, so we had not noticed this effect prior to the first observing run. This is the main reason the second run was during the summer: we hoped to avoid this problem. During the summer run, however, problems still occurred. While the devices did not shut off entirely, they appeared to become less sensitive with time. After acquiring a star and maximizing the count rate by moving the telescope and attempting to focus, the telescopes were left tracking with occasional touch-ups in position to maintain the count rate. However, over a period of $\sim 1 \mathrm{hr}$, a significant decrease in count rate was observed. Of course, since we had no imaging capability nor were we autoguiding, it is not possible to say with certainty that this problem was not with focus or telescope drift. However, we suspect temperature here as well, since at the start of the night, counts were within a factor of 2 of the predicted value based on target brightness and filter width, but as the night progressed (and temperatures became cooler), there was a general trend toward lower and lower count rates. This occurred at both telescopes in more or less the same way, which is at least some evidence that focus and telescope drift were not causing these effects.

Nonetheless, the raw data obtained in the June run are currently being analyzed for possible correlation peaks. An example is shown below in Fig. 3 . Here, the time-tagged photon stream from both detectors has been cross-correlated, but accounting for the change in timing difference expected from the change in sky position over the course of the observation. This changes the path length difference between the two channels and therefore the delay in time that correlated photons would arrive at the detectors. The site was assumed flat for this calculation, and the data from both channels were split into running "frames" where the maximum allowed 


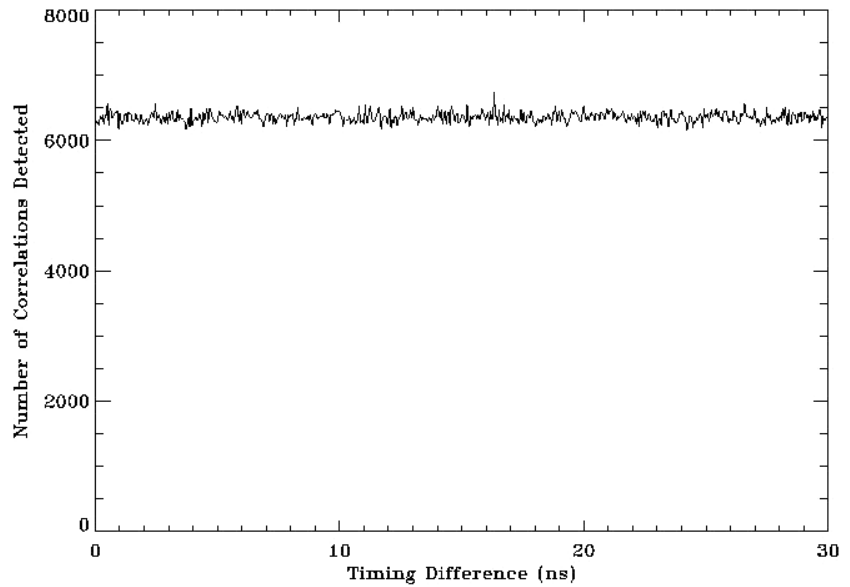

Fig. 3. Example of coadded timing correlation data accounting for change in the arrival time difference throughout the observation. The above represents approximately $1 \mathrm{hr}$ of data on Vega at Lowell. The data were taken through a $532-\mathrm{nm}$ filter that was $1 \mathrm{~nm}$ wide.

time delay was $0.5 \mu \mathrm{s}$ in length (which is much larger than the expected delay between the channels). The result shows total correlations as a function of timing difference when co-adding all frames. The cable length was longer from the signal correlator to the detector mounted on one of the two telescopes, and so we expected a delay due to this of $\sim 10 \mathrm{~ns}$. The most significant peak, which is not significant enough to claim the detection of a correlation signal, occurs at $16 \mathrm{~ns}$. There is another feature in the data near $10 \mathrm{~ns}$, which may be excess signal smeared over several tenths of a nanosecond. It is possible that this is related to a true correlation, but again the significance is not high enough to claim detection, and a mechanism for causing smearing on that timescale has not been identified. However, we are currently double-checking our analysis routines to make sure that the timing differences were correctly calculated. We plan to refine the reduction algorithms in the next year and attempt further observations after that. According to work previously done on this target reported by Hanbury Brown et al. (1967), the star is actually resolvable at a baseline of $\sim 50 \mathrm{~m}$, so that, depending on the precise geometry of the observation, the baseline may have been near the first minimum of the Airy pattern for the star, or near the secondary maximum. Thus, we would not expect a large signal in any case, though it could be that some data files making up our hour-long observation might show stronger signals than others, if the baseline shifted between these two cases during the hour. Further study will be required before more definitive statements can be made.

One of the main reasons to revisit intensity interferometry in our view is that it is possible to record the photon arrival times at the two stations independently and then correlate them after the fact. This permits the possibility of a wireless setup, where each station would have its own timing correlator and GPS timing card. Commercially available GPS cards give timing with accuracies of tens of nanoseconds. That alone is not sufficient for intensity interferometry, but if the stations could be roughly synchronized with this technology and the timing is stable over the integration time needed (which may impose a limit in integration time as small as the order of seconds), then the correlation signal could be searched for in the data stream over an interval determined by the uncertainty of the GPS cards (which should be less than 1 frame of data as analyzed above). Of course, there will be a further offset in the timing difference between photons arriving at each station from the site geometry and the sky position of the source, but these are measurable and predictable in principle. We hope to obtain a timing card and make tests of the stability in the near future to better understand the limit on integration time that may be imposed.

If a wireless version of this instrument can be built, then the advantage is that the baseline would no longer be limited by cable lengths or (in the case of amplitude-based long baseline optical interferometry) the length of optical beam paths. An instrument with a baseline of at least of order of one to a few kilometers could in theory be created simply by taking a SPAD and a timing module to telescopes separated by the desired distance. Returning to the three examples cited in the introduction, on the SCSU campus, a pair of large Dobsonian telescopes could be separated by 1-2 km. At Lowell, we are limited to a baseline of $\sim 50 \mathrm{~m}$. However, the 4-m Mayall Telescope and the $3.5-\mathrm{m}$ WIYN telescope are separated by $\sim 700 \mathrm{~m}$ at Kitt Peak; these could make for a very interesting intensity interferometer if used in combination. The hardware would essentially constitute a visitor instrument at each telescope, but when in operation, it would give unprecedented angular resolution in the visible. As we have shown, 6th magnitude stars could be observed at Kitt Peak in a reasonable amount of observing time. 


\section{Multi-Channel Light-Bucket Intensity Interferometer Arrays}

\subsection{Introduction}

Genet and Holenstein (2010) point out that, compared to a two-telescope, single optical channel per telescope intensity interferometer, the overall signal-to-noise ratio (SNR) of an intensity interferometer can be significantly increased by not only including many low cost, light bucket telescopes in a large array, but also by splitting the light on each telescope into multiple color channels. The gain in the array's SNR, as compared to a twotelescope, one channel interferometer, is to first approximation:

$\mathrm{SNR}_{\text {Array }}=\left[\left(\begin{array}{c}N_{\text {Array }} \\ 2\end{array}\right) M_{\text {Channels }}\right]^{1 / 2} \mathrm{SNR}_{\text {Baseline }}$

where $\mathrm{SNR}_{\text {Array }}$ is the overall SNR (gain) of an array of $N$ telescopes, each with $M$ channels, as

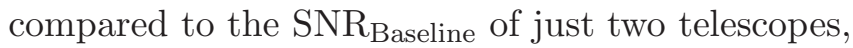
each with just a single channel. " $N$ choose 2 " is the number of baselines. For 10 telescopes, for instance, this is $(10 * 9 / 2)=45$ different baselines. For large $N$, Eq. (2) goes linearly with $N$, and therefore also linearly with total collection area. Similarly, the SNR for a single baseline scales with collection area, meaning that splitting larger apertures into smaller ones does not change the SNR. Increasing the number of telescopes in the array or the number of channels per telescope array, or both, will for the same integration time allow fainter magnitudes to be reached or, alternatively, will reduce the integration time for the same magnitude.

As the number of telescopes in an array becomes large, the improvement in SNR with respect to a two-telescope system is nearly directly proportional to the number of telescopes, $N$, i.e. there are no diminishing returns as telescopes are added to the array. On the other hand, adding additional channels on each telescope only improves with diminishing returns as $M^{1 / 2}$. A 9-channel system improves SNR by a factor of 3 , while a 100 channel system improves SNR by a factor of 10 .

The sensitivity of Hanbury Brown's intensity interferometer was similar to the two-telescope, single channel, Lowell Observatory curve shown above in Fig. 1. An array of seven Lowell-aperture telescopes would give 21 baselines instead of just one, and if each had 100 optical channels instead of one, the overall SNR would increase by about $(2100)^{1 / 2}$ or 45 times. This implies that the curves in Fig. 1 would translate to the right by about 4 magnitudes. Since each additional magnitude added allows one to observe $300 \%$ more stars, a four-magnitude shift of the curves to the right would enable one to observe over 250 times more program objects than a single baseline, single channel system.

We expect that the telescopes in intensity interferometry arrays will use low resolution, on-axis, essentially non-imaging light bucket optics, there being little reason to pay for high quality optics when they are not required. With somewhat large spot sizes in the focal plane, other telescope design parameters besides optics can also be relaxed, further reducing costs.

While photomultipliers are well-suited to light bucket operation, single SPAD have a small sensitive area in the focal plane that requires the use of imaging quality telescopes. However, SPAD arrays have been developed that overcome this smallsensor difficulty and in addition should - thanks to multiple, parallel sensors - reduce or eliminate the dead time inherent with single SPAD detectors.

Massimo Ghioni, Ivan Rech, and Angelo Gulantti at the SPAD Lab, Politecnico di Milano (Micro Photon Devices), have developed a $6 \times 8$ matrix of 50-micron SPAD elements on a 240$\mu \mathrm{m}$ pitch. Although the fill factor of this array is currently low, with the addition of a microlens array, this detector could be suitable for operation on light bucket telescopes. Further development could produce a much larger array. Please see http://www.everyphotoncounts.com/ for details.

For a given budget and state of technology, there should, at least in theory, be an optimal combination of the number of telescopes and channels per telescope that would maximize the SNR gain over a two-telescope, single channel baseline system. Because the two key technologies - low cost light bucket telescopes and multiple-channel high speed single photon sensors - are rapidly evolving, we will just consider technology development, leaving system optimization for later consideration. The costs that need to be considered during system design are not just the acquisition and installation costs, but also the long-term operating and maintenance $(\mathrm{O} \& \mathrm{M})$ costs.

Besides SNR and cost, spatial resolution the primary reason for considering intensity 
interferometry - is an important factor. As suggested above, modern digital optical intensity interferometry, similar to radio astronomy, can perform correlations remotely or after the fact. Thus, unlike amplitude interferometry, there are in theory no direct physical limitations. This opens up the possibility, as suggested by Joseph Ritter at the University of Hawaii's Institute for Astronomy, of space-based, light bucket intensity interferometry arrays with very large baselines and ultra-high resolution (Genet et al., 2013).

\subsection{Light bucket mirror and telescope developments}

An informal group, the Alt-Az Initiative, www. AltAzInitiative.org, has been developing low-cost meter-class mirrors and telescopes for the past six years (Genet et al., 2010). Three Initiative conferences and a number of workshops have been devoted to the development of low cost mirrors and telescopes. A significant portion of these development efforts have focused on the development of spincast epoxy, meniscus, and foam glass mirrors, as well as light bucket telescope structures and control systems.

The optical quality of non-imaging light bucket mirrors is evaluated somewhat differently than for imaging mirrors (Holenstein, Mitchell \& Koch, 2010). Specifically, what must be maximized in scientific studies is the SNR of the program object measures. For many types of observations, light bucket telescopes produce a superior SNR compared with conventional imaging telescopes of the same cost because they: (i) collect and deliver more photons to the detector thereby increasing the signal and reducing shot noise, (ii) have larger apertures, which reduce scintillation noise, and (iii) can be lightweight, which allows them to be transported to desirable observing locations and set up quickly. Stellar intensity interferometry can benefit from light bucket mirror and telescope technology since sub-arcsecond imaging of the program object is not needed. Instead, the moderately aberrated optics just need to produce a point spread function delivering most of the photons on the detector and excluding a moderate amount of the background.

Spin-cast mirrors take advantage of the natural tendency of a spinning liquid to assume a parabolic shape. Liquid mercury mirrors, such as used in the 6.5-m telescope at the University of British Columbia, have provided high quality images, although they are limited to observations near the zenith (Hickson et al., 1998). Spin-cast epoxy mirrors have suffered from edge effects, large temperature coefficients of expansion, and other problems, although organic chemist Lisa Brodhacker at Lander University has made considerable strides in perfecting this technology (Richardson \& Brodhacker, 2010). Because spin-cast epoxy mirrors are made from low cost materials and do not require optical grinding, polishing, or figuring, they hold the greatest promise for low cost.

Thin meniscus mirrors have worked well on telescopes as large as $8 \mathrm{~m}$. Numerous active actuators maintain the mirror's shape. Smaller aperture, very thin meniscus mirrors are being used in low cost light bucket telescopes. Connelley (2010) developed a very simple active optics system for a small, thin meniscus mirror that he is now applying to a thin 1-m meniscus mirror.

Aurigema and collaborators have developed a line of foam-glass composite mirrors that, after five years of development, have reached $1 \mathrm{~m}$ in aperture (Aurigema, Davis \& Genet, 2010). Two thin sheets of float glass - either soda lime or borosilicate form top and bottom plates that are fused onto a foam-glass core. Since the foam glass has a density that is just a small fraction of solid glass, and the glass plates are typically only $6 \mathrm{~mm}$ thick, the finished mirror only weighs a fraction of what a solid mirror would weigh, yet is just as stiff. A somewhat similar approach to making lightweight mirrors has been developed by Tong Liu at Hubble Optics. Thin top and bottom plates are fused to round, small diameter glass spacers between the plates.

Secondary mirrors, due to their convex shape, can be expensive to make. However, thanks to the lower optical requirements for light bucket secondary mirrors, single-point, diamond-turned mirrors can be made at low cost on high-speed, numerically-controlled lathes. Such mirrors can be made to any mathematically specified figure of revolution. Thus somewhat complex shapes, such as the secondary mirrors for Pressman-Carmichael optical systems, can be produced. The primary mirrors for Pressman-Carmichael optical systems are spherical, are relatively easy to produce, and hence low in cost, making these optical systems attractive for use in light bucket telescopes.

Very low cost, portable, meter-class telescopes have been developed over the past five years (Genet, Henden \& Holenstein, 2010). A 1.0-m portable 
telescope has seen initial operation. This telescope, which features a thin $(19 \mathrm{~mm})$ spherical primary mirror on an 18-point flotation system, has a simple mechanical primary mirror shaping system and a two-element spherical aberration correction system at prime focus. The telescope weighs less than $500 \mathrm{lbs}$. and can be transported in the back of a sports utility vehicle. A follow-on 1.0-m portable telescope is nearing completion and will be a test bed for both spin-cast epoxy and foam-glass 1-m mirrors.

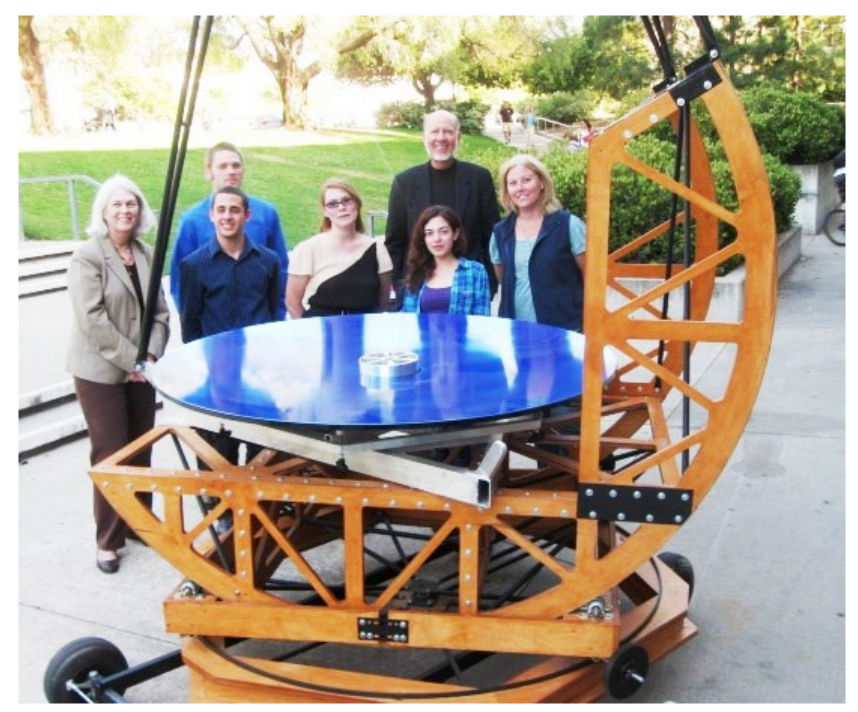

Fig. 4. Students (and faculty) at California Polytechnic State University stand behind the portable 1.5-m alt-az telescope they designed and built. The thin meniscus mirror (with a blue protective plastic coating) is supported by a 27-point Wiffletree.
A 1.5-m portable telescope, shown in Fig. 4, has been built and should see initial operation by late 2013. Finite element analysis (FEA) was used to maximize the structure's stiffness-to-weight ratio. The structural design features planar trusses automatically cut by a CNC mill with an eye toward low-cost mass production and assembly in a manner somewhat similar to Ikea furniture. The very thin $(19 \mathrm{~mm})$ mirror is supported axially by a 27 -point flotation system, and radially from its center-ofgravity by a spherical bearing which is actually in front of the front surface of the $\mathrm{f} / 2.5$ mirror. Adjustable magnetic forces are applied to the six outer Wiffletree triangles of the mirror support system to adjust the overall shape of the spherical mirror. A Sidereal Technology control system provides rapid and precise slewing and tracking.

The basic Planar truss design of the $1.5-\mathrm{m}$ portable telescope has been applied to the design of a 2.4-m portable telescope, the largest aperture that can be legally hauled down roads without special permits or packed in standard shipping containers. As with the 1.5-m telescope, the use of CNC-produced planar trusses should reduce construction and assembly costs. FEA analysis was used to optimize the structure's stiffness-to-weight ratio. CAD drawings were made for the telescope, and from these a conceptual visualization of an array was made as shown in Fig. 5 .

\subsection{Channels per telescope}

For systems using photomultipliers as the sensors, the incoming light can be split into a number

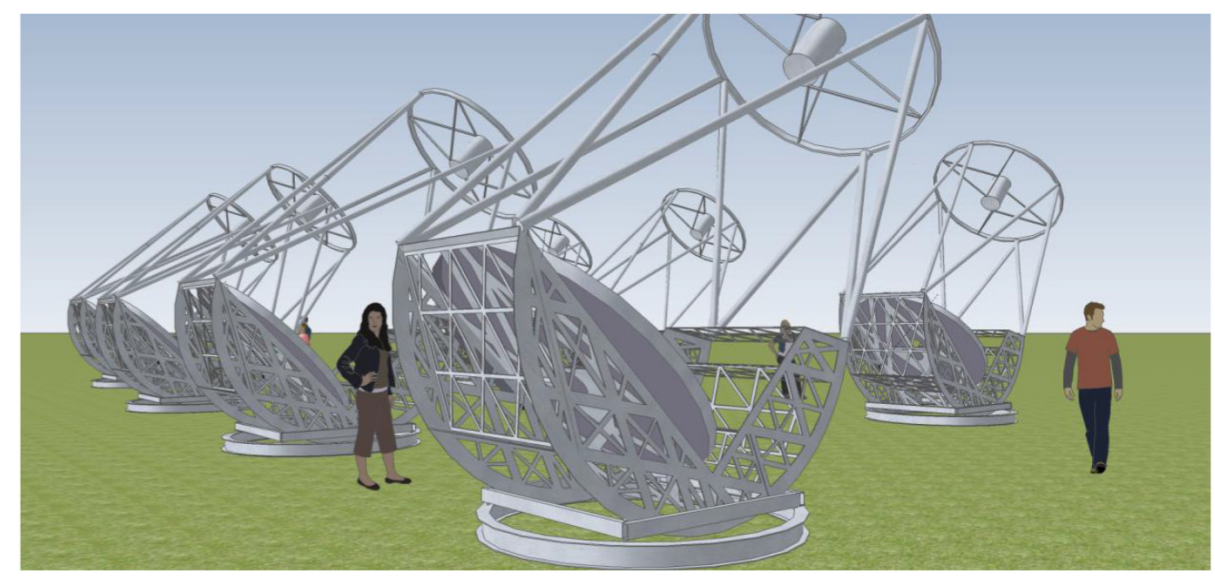

Fig. 5. Conceptual drawing of an array of lightweight, low cost, $2.4 \mathrm{~m}$ telescopes. The telescopes might feature a PressmanCarmichael optical system with a low cost, spherical primary mirror and a single-point, diamond turned secondary mirror. A 100-channel sensor on each telescope might feature an advanced SPAD array. 
of channels, perhaps 4 to 16 channels, by way of dichroic filters or other dispersing mechanisms, followed by narrowband interference filters. This would yield SNR gains, respectively, of 2 to 4 times compared to single channel systems. Adding multiple channels, once they are developed, may be less costly than adding additional telescopes.

Providing many channels, say 100, for an overall gain of 10 over a single channel, will probably require an area detector and a single dispersing element such as a grating. A much larger SPAD array than the one mentioned above might be suitable. Another intriguing possibility is the development of an EMCCD camera with the ability to handle very high data rates. While EMCCD cameras are currently capable of single photon detection, they cannot handle the high data rates required for intensity interferometry.

\subsection{OEBM costs and automation}

Initial acquisition and installation costs can, from a life cycle cost perspective, be less than the longrun operations and maintenance (O\&M) costs over the lifetime of a system. This is especially true for telescopes as they generally have long lifetimes. Perhaps the most direct way to reduce O\&M costs is full automation. Multiple automatic telescopes at fully robotic observatories have now been in continuous operation for over a quarter of a century (Genet, 2011), and the experience gained in their design, installation, and operation should apply to intensity interferometry arrays. A 2011 conference, Telescopes from Afar, provided an extensive review of the current state-of-the-art in automatic telescopes and robotic observatories. Conference papers are available at http://tfa.cfht.hawaii.edu/ and http://adswww.harvard.edu/.

Reliability and maintainability can be designed into systems to reduce O\&M costs. While this may raise the initial costs somewhat, it can pay off handsomely in the long run, especially when there are multiple identical telescopes and instruments.

\subsection{Space arrays}

Placing intensity interferometry telescope arrays in space could allow very long baselines between the individual telescopes (Genet et al., 2013). Space telescope mirrors have, of necessity, always been lightweight. They have also been of high optical quality and quite expensive. An array of low optical quality light bucket space telescopes has the potential of being relatively low cost.

An innovative technology is being developed by Joseph Ritter and Lisa Brodhacker for largeaperture, space-based, light bucket telescopes (Genet et al., 2013). Their technology uses optically active, thin-film material to construct ultralightweight mirrors. A laser activates the mirror's material to provide shape correction.

\section{Science}

Using the SPAD set-up at Lowell, we would like to pursue two main science goals. The first is the observation of bright spectroscopic binary stars. An instructive example is the spectroscopic binary $\alpha$ Aurigae (Capella), which is one of the brightest stars in the sky. Torres et al. (2009) have shown that an extremely precise analysis of this system does not yield agreement with stellar structure theory, and there may be a number of other systems in a similar situation. Looking at the ten brightest spectroscopic binaries observable in the Northern Hemisphere (including $\alpha$ Aur), the fractional error in semi-major axis listed in the Sixth Orbit Catalog of Visual Orbits has median value of $\sim 0.013$, only barely sufficient for meaningful comparisons with theory (Mason and Hartkopf, 2007; Andersen, 1991). Torres et al. were able to do such a detailed study of $\alpha$ Aur essentially because in that case the fractional error was much smaller, 0.001. This illustrates that the set-up at Lowell would be quite valuable in improving orbital elements of such systems to the sub-percent level.

The second area of interest is imaging of stellar surfaces. Intensity interferometry has not so far been proven in the imaging regime, where at least three stations would be required. However, Nuñez et al. (2012) have simulated this situation and proposed a Cauchy-Riemann-based algorithm to achieve imaging from intensity correlations. We expect to make a useful contribution to analyzing features of stellar surfaces at blue wavelengths. Stellar diameters obtained with current interferometers rely on spectral energy distribution models, but generally without interferometric measurements in the blue. Limb darkening models would be greatly helped with a larger coverage in wavelength, so we will be in a position to provide stringent constraints. Studies of star spots and magnetic activity on young stars would also be aided by more data in the blue. Since intensity 
interferometry does not lose signal-to-noise ratio as the band pass is narrowed, this presents the interesting possibility of obtaining extremely narrowband image information of stellar surfaces, in, for example, the sodium D-lines, with the same limiting magnitude as discussed above. A greater number of such studies would help us understand the evolution of massive stars and mass loss rates, as well as evolution of pre-main-sequence stars.

Further on the horizon, the very long baselines possible with wireless separated-station intensity interferometry discussed in Sec. 3 has the potential to open up entirely new vistas in astronomy. For a ground-based prototype, if the discussed advances from modern electronics enable sensitivity to $\mathrm{V}<9$, a 700-m baseline should be fairly straightforward to realize between the large apertures on Kitt Peak, or Subaru-Gemini on Mauna Kea. Such a demonstration could easily obtain the first direct measurement of the size of a white dwarf, Sirius B, at an angular size of $\sim 30$ microarcseconds ( $\sim 1$ Earth radius at $2.7 \mathrm{pc}$ ) (Holberg, Oswalt \& Barstow, 2012; Oswalt \& Sion, 2002). A few high-mass $\mathrm{X}$-ray binaries (e.g. SS Cyg-like systems) would be sufficiently bright and have reasonable separations of about tens of microarcseconds for exploration of these strong gravity laboratories by an intensity interferometer prototype (Hilliwig et al., 2004; Coughlin et al., 2010).

For a putative space-based facility enabled by wireless operation, with baselines up to $20,000 \mathrm{~km}$ and a sensitivity down to $\mathrm{V} \sim 20$ enabled by large gossamer apertures, further exotica present themselves for investigation, down to nano arcsecond scales. Direct distances to nearby galaxies in the Local Group should be possible by observing bright spectroscopic binaries. Dozens of X-ray binaries could be split and probed for relativistic orbital effects (Coughlin et al., 2010; Unwin et al., 2008). The very highest nearby strong gravity regime, that of the galactic center, could be monitored for lasttime-of-light material as it enters the black hole at Sgr A* (Vincent et al., 2011). Even the very granularity of space-time itself could be explored, by mapping the increasing decoherence of light for cosmological sources with increasing baseline, due to the effect of the fundamental Planck scale of spacetime smearing out fringes (Lieu et al., 2003).

Although these sorts of observations sound mildly fanciful, the key enabling technology is the simple extension of intensity interferometry to the wireless regime. As described here, however, this appears to be imminently possible.

\section{Conclusions}

We have presented a description of intensity interferometry with modern detectors and instrumentation. While modern electronics allows for meaningful astronomical observations to be taken with two $\sim 1-\mathrm{m}$ telescopes, the use of SPAD detectors has so far been challenging, due mainly to their small size and sensitivity variations observed at the telescope. Use of state-of-the-art photomultiplier tubes is also being attempted. If the technique can be demonstrated, then the next step would be to make the instrument wireless, meaning that very large baselines could be used, much larger than is currently possible with amplitude-based interferometers. Test data taken with our current instrumentation are currently under analysis.

Low cost, lightweight, portable, meter-class telescopes have been developed that should work well for intensity interferometry arrays. While not yet developed, multi-channel sensors on each telescope in an array could significantly increase the performance of intensity interferometers. Spacebased light bucket arrays could have unprecedented resolution.

\section{Acknowledgments}

We are very grateful for the assistance of Larry Wasserman and Otto Franz during our time at Lowell Observatory. We are also grateful for the work by Reed Estrada and Chris Estrada on the 1.0- and 1.5-m light bucket telescopes. California Polytechnic State University engineering students Mounir El-Koussa, Laura Rice, and Mike Vicary designed and built the structure for the $1.5-\mathrm{m}$ telescope. Donny Mott designed and built the primary mirror support system for the telescope, while Dan Gray donated the Sidereal Technology control system, and Mike Itz at Displays Optical Technologies donated the spherical primary mirror. Dave Rowe at PlaneWave Instruments and John Ridgely at California Polytechnic State University (Mechanical Engineering) provided invaluable technical advice on the telescope's design. The Collins Educational Foundation Gravic Inc. provided funding for the 2.4-m design. We thank Vera Wallen for reviewing this paper. 


\section{References}

Andersen, J., 1991, Astron. Astrophys. Rev., 3, 91.

Aurigema, A., Davis, D. \& Genet, R., 2010, Glass foam composite mirror developments. In Genet, R., Johnson, J. \& Wallen, V., eds., The Alt-Az Initiative: Telescope, Mirror, \& Instrument Developments, Santa Margarita, CA: Collins Foundation Press.

Connelley, M., 2010, Active optics for thin meniscus telescope mirrors. In Genet, R., Johnson, J. \& Wallen, V., eds., The Alt-Az Initiative: Telescope, Mirror, 8 Instrument Developments, Santa Margarita, CA: Collins Foundation Press.

Coughlin, J. L., Gelino, D. M., Harrison, T. E. et al., 2010, ApJ, 717, 776 .

Genet, R., 2011, Telescopes from Afar, in Society for Astronomical Sciences, 30th Annual Symposium, 25-36.

Genet, Henden \& Holenstein, 2010, Light Bucket Astronomy, in Society for Astronomical Sciences, 29th Annual Symposium, 117-122.

Genet, R. \& Holenstein, B., 2010, Alt-Az light bucket astronomy. In Genet, R., Johnson, J. \& Wallen, V., eds., The Alt-Az Initiative: Telescope, Mirror, $\&$ Instrument Developments, Santa Margarita, CA: Collins Foundation Press.

Genet, R. et al., 2010, The Small Research Telescope Challenge, National Academies of Science Astro2010, The Astronomy and Astrophysics Decadal Survey, Position Paper 17.

Genet, R. et al., 2013, Earth- and Space-Based Light Bucket Intensity Interferometry Arrays, in Society for Astronomical Sciences, 32nd Annual Symposium, 157-167.

Hanbury Brown, R. \& Twiss, R. Q., 1956, Nature, 177, 27.

Hanbury Brown, R., Davis, J. \& Allen, L. R., 1974, MNRAS, 167, 121.

Hanbury Brown, R., Davis, J., Allen, L. R. \& Rome, J. M., 1967, MNRAS, 137, 393.

Hickson, P. et al., 1998, Proc. SPIE, 3352, 226.

Hilliwig, T. C., Gies, D. R., Huang, W. et al., 2004, ApJ, 615, 422 .
Holberg, J. B., Oswalt, T. D. \& Barstow, M. A., 2012, AJ, $143,68$.

Holberg, J. B., Oswalt, T. D. \& Sion, E. M., 2002, ApJ, 571, 512.

Holenstein, B., Mitchell, R. \& Koch, R., 2010, Figures of merit for light bucket mirrors. In Genet, R., Johnson, J. \& Wallen, V., eds., The Alt-Az Initiative: Telescope, Mirror, 8 Instrument Developments, Santa Margarita, CA: Collins Foundation Press.

Horch, E. P. \& Camarata, M. A., 2012, Proc. SPIE, 8445, $2 \mathrm{~L}$.

Klein, I., Guelman, M. \& Lipson, S. G., 2007, Applied Optics, 46, 4237.

Lebohec, S., Adams, B., Bond, I. et al., 2010, Proc. SPIE, $\mathbf{7 7 3 4}, 1$.

Lieu, R. \& Hillman, L. W., 2003, ApJ, 585, L77.

Mason, B. D. \& Hartkopf, W. I., 2007, in Binary Stars as Critical Tools 8 Tests in Contemporary Astrophysics, Proceedings of IAU Symposium \#240, eds. Hartkopf, W. I., Guinan, E. F. and Harmanec, P., p. 575.

Nuñez, P. D., Holmes, R., Kieda, D. \& LeBohec, S., 2012, MNRAS, 419, 172.

Radhakrishnan, V., 1999, in Synthesis Imaging in Radio Astronomy II, eds. Taylor, G. B., Carilli, C. L. \& Perley, R. A., ASP Conference Series, Vol. 180, 1999, p. 671.

Richardson, T. \& Brodhacker, L., 2010, Spinning the way to a perfect figure: Spin-cast polymer mirrors. In Genet, R., Johnson, J. \& Wallen, V., eds., The Alt-Az Initiative: Telescope, Mirror, $\&$ Instrument Developments, Santa Margarita, CA: Collins Foundation Press.

Shao, M. \& Staelin, D. H., 1977, JOSA, 67, 81.

Shao, M., Colavita, M. M., Staelin, D. H. et al., 1986, JOSA A, 3, P18.

Torres, G., Claret, A. \& Young, P. A., 2009, ApJ, 700, 1349.

Unwin, S. C., Shao, M., Tanner, A. M. et al., 2008, PASP, $\mathbf{1 2 0}, 863$.

Vincent, R. H., Paumard, T., Perrin, G. et al., 2011, MNRAS, 412, 2653. 